\title{
Polarized hydrogen emission lines in Mira stars: a mystery behind the shock
}

\author{
Nicolas Fabas ${ }^{1}$, Agnès Lèbre ${ }^{1}$ and Denis Gillet ${ }^{2}$ \\ ${ }^{1}$ GRAAL, UMR 5024,Université Montpellier II/CNRS, France \\ ${ }^{2}$ Observatoire de Haute-Provence, USR 2207, OAMP/CNRS, France
}

\begin{abstract}
We present a full spectropolarimetric study (in the Stokes parameters I, Q, U and $\mathrm{V}$ ) on omicron Ceti (the prototype of Mira stars), and focus on the strong polarization detected in Balmer emission lines. This study is made with observations from NARVAL instrument at TBL (Telescope Bernard Lyot) in Pic du Midi Observatory, France.
\end{abstract}

Keywords. Shock waves - AGB and post-AGB - line: formation

\section{Introduction}

Mira stars are cool, evolved and variable stars, the prototype being omicron Ceti (as known as Mira). They are radially pulsating stars with long period of luminosity (e.g., the period of o Ceti is 332 days). From maximum to minimum light, the spectral type of o Ceti varies from M5 to M9. A cool and very extended stellar atmosphere is present, which is typical from this kind of late-type stars. Those atmospheres are surrounded with a circumstellar envelope. Among the peculiar features of Mira stars' spectra, emission lines are detected. The hydrogen Balmer lines are observed in emission during about $80 \%$ of the luminosity period and are supposed to be formed in the radiative wake of a hypersonic shock wave propagating periodically throughout the stellar atmosphere (Fadeyev \& Gillet, 2004).

\section{Spectropolarimetric analysis}

Spectro-polarimetric signatures are detected in the 4 Stokes parameters (IQUV) associated to Balmer hydrogen lines (from $H \alpha$ to $H \delta$ ). Those signatures appear to be time variable, being much more visible and structured at the maximum luminosity, when the shock is emerging from the photosphere and is propagating with a high intensity. Below (Fig. 1) are presented the signatures for $H \beta$ and $H \delta$ at the three observed phases $(\phi=0.58$, 1.00 and 1.06). From Stokes parameters Q \& U, linear polarization in the Balmer lines can be estimated (Fig. 2).

\section{Results}

We confirm the earlier and single detection in the $H \beta$ line (McLean \& Coyne, 1978) on o Ceti when observed at its maximum luminosity: Balmer hydrogen emission lines are strongly polarized, much more than the local continuum. Besides, the linear polarization rate, in all the Balmer lines, appears to be time variable, and null at the minimum light. We find that this polarization in Balmer emission lines is likely to be linked with the emission line formation mechanism occurring within the de-excitation post shock wake of the strong shock wave propagating throughout the stellar atmosphere at each pulsation 

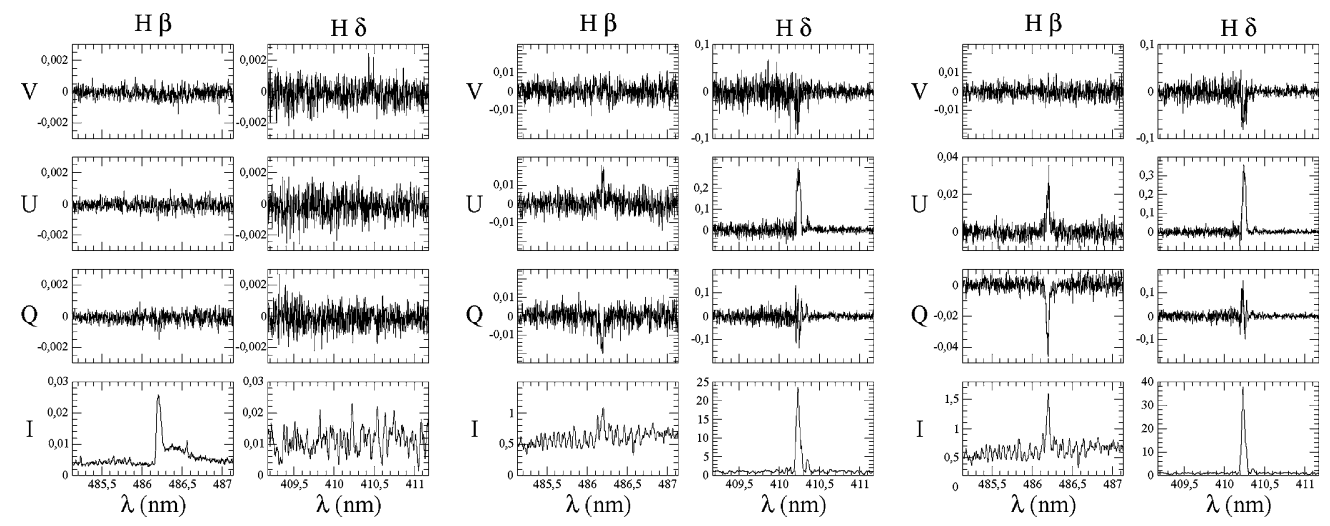

Figure 1. Signatures for $H \beta$ and $H \delta$ at the three observed phases ( $\phi=0.58,1.00$ and 1.06)

$\mathrm{H} \beta$

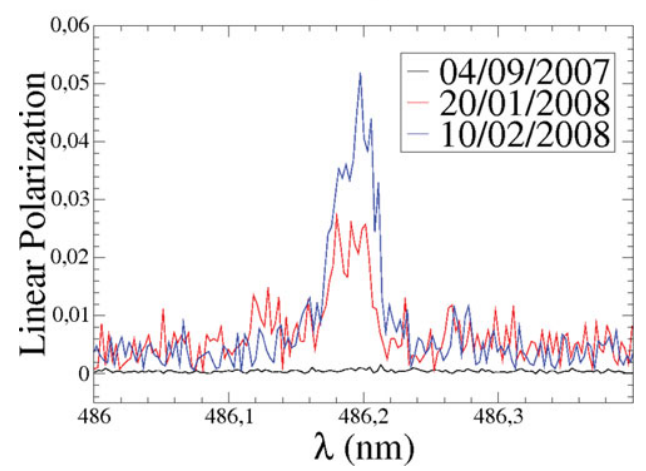

Figure 2. Linear polarization for $H \beta$ at the three observed phases $(\phi=0.58,1.00$ and 1.06)

cycle. Moreover, according to shock wave theory, an electromagnetic field should appear just behind the shock front (where emission lines are formed), inducing polarization. To date, this field has never been conclusively detected on Mira stars (neither on any other type of radially pulsating star). Our spectro-polarimetric observations constitute the first attempt to fully characterize and understand the associated physical mechanism. Eventually, this study would be also useful for the characterization of the shock wave with respect to the central star.

\section{Acknowledgements}

N. Fabas would like to thank the IAU for the grant he was given to take part to IAU Symposium 259.

\section{References}

Fadeyev, Y. A. \& Gillet, D. 2004, A\&A 420, 423

McLean, I. S. \& Coyne G. V. 1978, ApJ 226, 145 